\title{
Summary of Observational Aspects
}

\author{
C. D. Scarfe \\ Department of Physics and Astronomy, University of Victoria, \\ Victoria, B.C. V8W 3P6, Canada \\ email: scarfe@uvic.ca
}

\begin{abstract}
This summary covers the first two days of the Symposium, in which the oral papers were primarily observational in nature. It also mentions several of the many observational posters associated with those discussions.
\end{abstract}

Keywords. (stars:) binaries: general

\section{Introduction}

First, let me thank my colleagues on the SOC for giving me the opportunity to attempt to summarize the first portion of this meeting, and with it the incentive to listen carefully to several interesting invited papers, and also to read numerous posters covering a wide range of topics relevant to binary and multiple stars. So much information has been presented at this meeting that I am very grateful not to have to cover it all, but to leave part of it in the very capable hands of Virginia Trimble, who will speak next. We have considered how we might best share our pleasant duty, and have settled on the following: she will cover the primarily theoretical papers of the final day and a half, and I am to deal with the earlier portion, as stated above.

One of the few advantages of growing older is that one can remember a goodly number of interesting meetings. For myself, I can declare that the most interesting of all have been IAU-sponsored, and concerned primarily with binary stars. Some of those that I have enjoyed include Colloquium 5 in Nice, France, in 1969, Colloquium 33 on Multiple Stars, in Oaxtepec, Mexico, in 1976, Colloquium 62 on Current Techniques, in Flagstaff, U.S.A. in 1981, Colloquium 107 on Algols, in Victoria, Canada, in 1988, Colloquium 135 on Complementary Approaches, near Atlanta, U.S.A., in 1992, Symposium 200 on Formation of Binary Stars, in Potsdam, Germany, in 2000, Colloquium 191 on their Environment and Evolution, in Merida, Mexico, in 2003, and finally the present one, Symposium 240. Most of those meetings have been Colloquia, a series whose discontinuance is regrettable because it leaves a gap in the range of meeting size and of breadth of topic which the IAU offers its members.

\section{Oral Papers}

Most of the above-mentioned meetings have included papers on a range of topics scattered around the stated theme of the meeting, and this one is no exception. However it seemed to me that at least early in the meeting the 'contemporary astrophysics' addressed by the speakers was mainly that of the binaries themselves, rather than wider applications.

Nevertheless the presentations contained much of interest, as presaged in the 'trailer' presented very lucidly at the beginning by Guinan (see Guinan et al. 2007). We began by learning about major developments in instrumentation for studying all sorts of binaries. 
Developments in long-baseline interferometry in the northern and southern hemispheres were summarized with great clarity by McAlister (2007) and Davis (2007) respectively, and Shao (2007) envisaged doing even better from space. Then Balega described the history of speckle interferometry. He reminded us of the vast flood of good speckle observations that began in the 1970s; we await eagerly a similar flood from long-baseline interferometry.

Next, Hatzes reminded us of the rapid progress in the precision of relative radialvelocity data, with wonderfully stable instruments that have left absolute radial-velocity accuracy far behind. But for many of us this is of little concern. Absolute velocities have limited uses for binary-star studies. Stability from night to night and over the long term is of greater value.

The use of large telescopes, and concentration upon spectroscopic observations, was urged by Ribas (2007), and Bonanos (2007) described the vast data banks derived from microlensing programs. In a comment, Wilson warned about the poor quality of some mass-produced light curves. Another speaker claimed that masses can be obtained from light curves without resort to spectroscopic data, but Leung (2007) indicates that this may not be true except in favourable cases, e.g., total eclipses in contact systems.

The prodigious efforts that have been made to extend the Washington Double Star Catalog, a valuable resource indeed, were described by Mason (2007). Then Hartkopf (2007) discussed the continued development of multiplicity nomenclature. However, it may not be easy always to apply that nomenclature in a way that does not require changes with time, as a brief consideration of the discovery sequence described by Harmanec et al. (2007) will reveal.

New and sophisticated methods of obtaining radial velocities were described by Hensberge \& Pavlovski (2007), but Parimucha \& Škoda (2007) found that the old method of matching profiles of individual lines with their mirror images produces better results in some situations.

On the following day, Peters (2007) described some remarkable spectroscopic observations of Algols, mostly in the far ultraviolet, and their interpretation in terms of models of the circumstellar material around the mass-gaining stars in those systems. Some information may be gleaned on the systems' past history by determination of the chemical composition of those materials. Then Kafka (2007) indicated how the pattern of accretion in cataclysmic variables depends upon their magnetic properties, and went into detail about what may be observed in the low states of VY Scl and AM Her systems, when the bright light of obscuring material is greatly reduced.

A beautifully clear introduction to tomography was presented by Richards (2007). She discussed the importance of eliminating stellar light to see more clearly the structure of disks (or is 'disk' a too simple description?!), and outlined the difficulties of interpreting the data in terms of models of that structure. Then Strassmeier (2007) gave a fine account of the challenge of mapping spotted stars, with numerous examples, and of the complexities arising if one tries to model the related magnetic fields by Zeeman-Doppler imaging. Then followed a most entertaining account by ten Brummelaar (2007) of the snags and pitfalls of long-baseline interferometry.

Wilson (2007) described the incorporation of various effects into modelling codes. This has been enabled by spectacular increases in computer speed and memory capacity, and have made the models' properties resemble those of real stars increasingly closely. Moreover, one can now archive very many model light curves, to provide rapid matching to the multitude of observed light curves that are becoming available, and to the even greater multitudes that are promised before long. 
The six final oral papers that fell under my purview were all concerned with large-scale surveys. Pourbaix described several potential automatic methods of identifying binaries and discovering some of their characteristics. Then Prša \& Zwitter (2007) discussed pipeline reduction of binary light curves, which will become essential if large-scale surveys from space discover binaries in the huge numbers expected. Koch et al. (2007) followed this with a very practical and straightforward account of the Kepler mission, whose purpose is to observe a single area repeatedly, to seek transits of earth-sized planets across their stars' disks, no small task considering that such an event may be manifested by a $0.1 \mathrm{mmag}$ decrease in light, that lasts for perhaps twelve hours and occurs once a year. It seems likely that objects such as eclipsing binaries will be found much more easily. Niarchos et al. (2007) gave us details of some important simulations, preparatory for handling the vast amounts of data anticipated from GAIA. Of course, if the numbers of binaries found by Mazeh et al. (2007) prove to be normal, those data will not be so numerous as supposed, by perhaps one order of magnitude. But that will still be a huge amount of data! Finally, Zwitter described a ground-based project to obtain a million radial velocities. This alone will keep the bibliography team busy for some time. One gets an impression from these various projects of something of a trade-off between wonderfully precise data in large but manageable quantities, and an overwhelming quantity of less precise data. The first of these - the manageable quantities of excellent data - makes me wish I were young again. The other makes me not so sure of this.

[Editors' note: Unfortunately the talks by Balega, Hatzes, Pourbaix and Zwitter mentioned above were not submitted to these proceedings.]

\section{The Posters}

I would now like to say a little about the posters, only a very few of which I have mentioned so far. They are wonderfully numerous and diverse, even though not all of the 181 listed in the abstract book actually appeared - I counted about 140. I spent four interesting lunch periods trying to read them, and although I did not read every word (some of them being very lengthy and detailed for posters), I did at least look at them all. Even so I cannot hope to mention them all here and I hope the authors of those I omit will forgive me. But it is important that I spend some time on them because I saw only a few participants up in the poster hall reading them for themselves. The poster hall's remote location was partly to blame for this, of course.

Some people said to me that the talks were too much on instruments, techniques and projects, and too little about science, and I have to agree, at least to some extent. However, that defect, if it was one, could be remedied by reference to the posters, where science was more predominant. That science covers a broad spread of objects, from common-proper-motion pairs (Kiselev et al. 2007, Sinachopoulos et al. 2007, Halbwachs et al. 2007, Johnston et al. 2007) to ultra-compact binaries in which a white dwarf is losing matter to its neutron-star companion (Nelemans 2007). It also ranges from massive O-type systems (Fernández Lajús \& Niemela 2007, Iping et al. 2007, Nesslinger et al. 2007) to brown dwarf pairs (Golimowski et al. 2007, Gómez Maqueo Chew et al. 2007), with total masses respectively 100 and 90 times that of Jupiter.

To me it seems not long since the discovery of V471 Tau, one of the first known binaries containing a non-interacting white dwarf and main-sequence star. But now several are known, and we heard in one of the posters selected for oral presentation, van den Besselaar et al. (2007), about a new object of that type, DE CVn. It is worth noting, however, that a second poster, Goker \& Tas (2007), presents similar but by no means identical parameters for the same system. 
As editor-in-chief of Commission 42's semiannual bibliography I am very much aware that certain binaries attract a lot of attention, and are the subject of many papers. That is also true among the posters at this meeting. For example, Cygnus X-1 is the subject of at least three, (Karitskaya et al. 2007a, 2007b, 2007c), by different members of the same group. As well, $\eta$ Carinae is the subject of at least two, Falceta-Gonçalves et al. (2007) and Gull (2007). Several old friends among the stars are also the subject of new work, including RZ Cas (Golovin \& Pavlenko 2007), $\epsilon$ Aur (Stencel 2007), AM Her (Kalomeni et al. 2007) and $\beta$ Lyr (Chadima et al. 2007). It is nice to see that V1500 Cyg, once known as the fastest nova in the Galaxy, is still misbehaving almost 30 years after it first attracted widespread attention. Also, of course, RS Oph is active once again (Stringfellow et al. 2007).

Some of the posters describe quite basic results, which might be regarded as not quite at the cutting edge. But in most cases they are authored by people who do not have access to modern equipment, or who lack financial support in their home countries, or both. And in general they serve a useful purpose in filling in gaps in our knowledge. Their authors are to be congratulated on their heroic efforts, and applauded for their contributions. (Those of us who do have better support may well not appreciate how lucky we are!) So too should we congratulate the amateurs, who have long contributed much to the study of variable stars, including eclipsing binaries, and are now contributing to visual binary work as well. Examples include Agati et al. (2007) and Rica et al. (2007).

A few further posters that I would like to mention include those of Liu \& Qian (2007), who propose an imaginative design for a telescope, Kövári et al. (2007), who warn of aliases that may interfere with the interpretation of Doppler imaging, Scardia et al. (2007), who describe some beautiful work on $\gamma$ Vir, which has just passed its only periastron in any of our lifetimes, and Lampens et al. (2007), who present new work on $\theta^{2} \mathrm{Tau}$, a binary both of whose components pulsate, and which lies at the top of the Hyades' main sequence.

There are more posters, in addition to those already mentioned, on a wide variety of topics, such as:

- 1. Chromospherically active binaries (Korhonen \& Järvinen 2007, Gálvez et al. 2007 and $\mathrm{Gu}$ et al. 2007),

- 2. Symbiotic stars (Hric et al. 2007),

- 3. Dwarf novae (Hourihane et al. 2007, Viallet \& Hameury 2007),

- 4. Binaries with pulsating components (Latković 2007 and Evans et al. 2007),

- 5. Binaries in clusters (Southworth 2007, as well as Hourihane et al. 2007, mentioned above), and

- 6. Data mining (Lee et al. 2007 and Devor \& Charbonneau 2007).

One poster that particularly interests me is that of Amado (2007), who describes the use of binaries to extend a modified version of the Barnes-Evans relationship to low masses.

Many of the posters are straightforward descriptions of interesting systems, but a significant number do indeed live up to the Symposium's title, providing applications to astrophysics in general. The range of topics is breathtaking. This could be regarded as a measure of the Symposium's lack of focus, or more charitably of its inclusiveness. The choice is yours.

\section{Some Further Thoughts}

Quite a lot has been said, and written, here about obtaining reliable masses and radii from double-lined eclipsing binaries, and of getting indirect distance estimates for such 
objects, by incorporating effective temperature information. I say indirect, because this always requires a calibration of effective temperature against colour index or spectral type.

Several other contributors, e.g., Fekel \& Tomkin (2007), have mentioned deriving masses and distances from visual-spectroscopic systems, which yield so-called orbital parallaxes. These have proved to be much more accurate than Hipparcos parallaxes, and have indeed served as useful checks on the latter. Very often they agree well, but in some cases there is pronounced disagreement, which is usually interpreted in terms of vitiation of the Hipparcos result by the binarity itself. It is curious that this does not happen more consistently. The region containing binaries for which such combined observations are possible is still a small one near the sun; long-baseline interferometry should make it much larger.

However, remarkably little has been said about the pleasant prospect of overdetermining the astrophysical quantities, by interferometric resolution of binaries that are also double-lined and eclipsing. One such object is $\gamma$ Per (Griffin 2007), whose eclipses, discovered in 1990 (Griffin 1992), were of sufficient interest at Colloquium 135 that the system was featured on the cover of that meeting's Proceedings, although the interferometric aspect of the system did not feature prominently in this meeting's poster presentation.

Such a combination of observations would provide distances for eclipsing systems independently of any calibration of colour against temperature, and with the use of observed spectral energy distributions, would permit direct deduction of effective temperatures from absolute bolometric luminosities and radii. We could then provide other astrophysicists with an absolute calibration of colour indices and bolometric corrections against effective temperatures, surely a worthy goal in view of this Symposium's avowed theme.

It will not be easy to achieve that goal. Of course one needs to choose well-separated eclipsing pairs, of which many are known. But those on the lower main sequence are faint and inaccessible to interferometry, and those on the upper main sequence are distant, and hence also inaccessible. So we will have to build outward from solar-type stars towards those both hotter and cooler, as the capability of interferometry increases.

The most stringent tests of stellar models also require chemical information for each component of a binary. Spectral disentangling is now giving us a way forward to reach this goal. It is the subject here of several posters, e.g., Pavlovski \& Tamajo (2007), Hadrava (2007), and Gerbaldi \& Faraggiana (2007), as well as of the talk by Hensberge \& Pavlovski (2007). Ages are also valuable, and these can sometimes be obtained for field main-sequence stars in wide binaries if their companion is a white dwarf, as discussed by Oswalt (2007).

I could go on, and mention yet more interesting posters, but I think it is now time to turn the floor over to Virginia Trimble.

\section{References}

Amado, P.J. 2007, these proceedings, 327

Agati, J.L., Caille, S., Debackere, A., Durand, P., Losse, F., Mantle, R., Mauroy, F., Mauroy, P., Morlet, A.G., Pinlou, C., Salaman, M., Soule, E.J., Thorel, Y., \& Thorel, J.C. 2007, these proceedings, 117

Bonanos, A.Z. 2007, These Proceedings, 79

Chadima, P., Ak, H., Harmanec, P., Demirçan, O., Yang, S., Koubský, P., Škoda, P., Šlechta, M., Wolf, M., Božić, H., Ruždjak, D., \& Sudar, D. 2007, These Proceedings, 205

Davis, J. 2007, These Proceedings, 45

Devor, J., \& Charbonneau, D. 2007, These Proceedings, 208 
Evans, N.R., Schaefer, G., Bond, H.E., Nelan, E., Bono, G., Karovska, M., Wolk, S., Sasselov. D., Guinan, E., Engle, S., Schlegel, E., \& Mason, B. 2007, These Proceedings, 102

Falceta-Gonçalves, D., Abraham, Z., \& Jatenco-Pereira, V. 2007, These Proceedings, 198

Fekel, F.C. \& Tomkin, J. 2007, These Proceedings, 59

Fernández Lajús, E. \& Niemela, V. 2007, These Proceedings, 120

Gálvez, M.C., Montes, D., Fernández-Figueroa, M.J., De Castro, E., \& Cornide, M. 2007, These Proceedings, 461

Gerbaldi, M. \& Faraggiana, R. 2007, These Proceedings, 120

Goker, U.D., \& Tas, G. 2007, These Proceedings, 128

Golimowski, D.A., Minniti, D., Henry, T.J., \& Ford, H.C. 2007, These Proceedings, 329

Golovin, A. \& Pavlenko, E. 2007, These Proceedings, 330

Gómez Maqueo Chew, Y., Stassun, K.G., Mathieu, R., \& Vaz, L.P., 2007, These Proceedings, 330

Griffin, R.E.M. 2007, These Proceedings, 333

Griffin, R.F. 1992, in: H.A. McAlister \& W.I. Hartkopf (eds.) Complementary Approaches to Double and Multiple Star Research, IAU Colloquium 135 (San Francisco: ASP Conf. Ser.), vol. 32 , p. 98

Gu, S.-H., Cameron, A.C., Barnes, J.R., \& Yang, J.-Y. 2007, These Proceedings, 461

Guiman, E., Harmance, P. \& Hartkopf, W. 2007, These Proceedings, 5

Gull, T.R. Nielsen, K.E., Corcoran, M.F., Hillier, D.J., \& Homaguchi, K. 2007, These Proceedings, 387

Hadrava, P. 2007, These Proceedings, 111

Halbwachs, J.-L., Mayor, M., \& Udry, S. 2007, These Proceedings, 427

Harmanec, P., Mayer, P., Božić, H., Eenens, P., Guinan, E.F., McCook, G., Koubský, P., Ruždjak, D., Sudar, D., Šlechta, M., Wolf, M., \& Yang, S. 2007, These Proceedings, 64

Hartkopf, W.I. 2007, These Proceedings, 97

Hensberge, H. \& Pavlovski, K. 2007, These Proceedings, 136

Hourihane, A.P., Callanan, P.J., \& Cool, A.M. 2007, These Proceedings, 117

Hric, L., Gális, R., \& S̆melcer, L. 2007, These Proceedings, 387

Johnston, K., Oswalt, T., \& Valls-Gabaud, D. 2007, These Proceedings, 429

Kafka, S. 2007, These Proceedings, 154

Kalomeni, B., Yakut, K., \& Pekunlu, E.R. 2007, These Proceedings, 123

Karitskaya, E.A., Agafonov, M.I., Bochkarev, N.G., Bondar, A.V., \& Sharova, O.I. 2007, These Proceedings, 65

Karitskaya, E.A., Lyuty, V.M., Bochkarev, N.G., Shimanskii, V.V., Tarasov, A.E., Galazutdinov, G.A., \& Lee, B.-C. 2007, These Proceedings, 122

Karitskaya, E.A., Shimanskii, V.V., Bochkarev, N.G., Sakhibullin, N.A., Galazutdinov, G.A., \& Lee, B.-C. 2007, These Proceedings, 130

Kiselev, A.A., Kiyaeva, O.V., \& Izmailov, I.S. 2007, These Proceedings, 129

Koch, D., Borucki, W., Basri, G., Brown, T., Caldwell, D., Christensen-Dalsgaard, J., Cochran, W., De Vore, E., Dunham, E., Gautier, T., Geary, J., Gilliland, R., Gould, A., Jenkins, J., Kondo, Y., Latham, D., Lissauer, J., \& Monet, D. 2007, These Proceedings, 236

Korhonen, H. \& Järvinen, S.P. 2007, These Proceedings, 453

Kövári, Zs., Bartus, J., Oláh, Strassmeier, K.G., Rice, J.B., Weber, M., \& Forgács-Dajka, E. 2007, These Proceedings, 212

Lampens, P., Frémat, Y., De Cat, P., \& Hensberge, H. 2007, These Proceedings, p. (poster 125)

Latković, O. 2007, These Proceedings, 460

Lee, K.-W., Lee, B.-C., \& Park, M.-G. 2007, These Proceedings, 266

Leung, K.C. 2007, These Proceedings, 124

Liu, Z. \& Qian, S.B. 2007, These Proceedings, 64

Mason, B.D. 2007, These Proceedings, 88

Mazeh, T., Tamuz, O., \& North, P. 2007, These Proceedings, 230

McAlister, H.A. 2007, These Proceedings, 35

Nelemans, G. 2007, These Proceedings, 124 
Nesslinger, S., Drechsel, H., Lorenz, R., Harmanec, P., Mayer, P., \& Wolf, M. 2007, These Proceedings, 326

Niarchos, P.G., Munari, U., \& Zwitter, T. 2007, These Proceedings, 244

Oswalt, T.D., Johnston, K.B., Rudkin, M., Vaccaro, T., \& Valls-Gabaud, D. 2007, These Proceedings, 300

Parimucha, S. \& Škoda, P. 2007, These Proceedings, 62

Pavlovski, K. \& Tamajo, E. 2007, These Proceedings, 209

Peters, G.J. 2007, These Proceedings, 148

Prša, A. \& Zwitter, T. 2007, These Proceedings, 217

Ribas, I. 2007, These Proceedings, 69

Rica, F.M., Benavides, R., \& Masa, E. 2007, These Proceedings, 121

Richards, M. 2007, These Proceedings, 160

Scardia, M., Argyle, R.W., Prieur, J.-L., Pansecchi, L., Basso, S., Law, N., \& Mackay, C. 2007, These Proceedings, 132

Shao, M. 2007, These Proceedings, 54

Sinachopoulos, D., Gavras, P., Medupe, Th., Ducourant, Ch., \& Dionatos, O. 2007, These Proceedings, 264

Stencel, R.E. 2007, These Proceedings, 202

Southworth, J. 2007, These Proceedings, 264

Strassmeier, K.G. 2007, These Proceedings, 170

Stringfellow, G.S., Walter, F.M., Wallerstein, G., York, D.G., Dembicky, J., Ketzebach, B., \& McMillan, R.J. 2007, These Proceedings, 386

ten Brummelaar, T. 2007, These Proceedings, 178

van den Besselaar, E.J.M., Greimel. R., Morales-Rueda, L., Nelemans, G., Thorstensen, J.R., Marsh, T.R., Dhillon, V., Robb, R.M., Balam, D.D., Guenther, E.W., Kemp, J., Augusteijn, T., \& Groot, P.J. 2007, These Proceedings, 105

Viallet, M. \& Hameury, J.M. 2007, These Proceedings, 212

Wilson, R.E. 2007, These Proceedings, 188 